\title{
From object to process; conceptual matrices in architecture's expanded field
}

\section{SIGRADI2018 TECHNOPOLITICAS \\ xxii congresso da sociedade iberoamericana de gráfica digital 22th conference of the iberoamerican society of digital graphics $07|08| 09$ |novembro|2018 iau usp | são carlos | sp br}

\author{
Adriana R. R. Lima \\ Faculty of Architecture and Urbanism, University of Sao Paulo | Brazil | adrianarrl@usp.br
}

Rafael A. C. Perrone

Faculty of Architecture and Urbanism, University of Sao Paulo | Brazil |

racperrone@gmanil.com

\begin{abstract}
The article focuses on the development of a diagrammatic method of design processes based on the elaboration of conceptual matrices that embrace the reading of some of Zaha Hadid 's design projects. The object of study is approached through its formative process, considering its continuous evolution, in a dynamic field of interactions and mediations. The architectural works studied, generated several diagrams through the research of their 'formative concepts' (Clark \& Pause)', later defined, as 'operative categories'. The definition follows the understanding of the architect's work as non-linear sequences of projective actions. The project's study, found similar strategies, which are generated as projective series, through similar spatial expressions and methods of conception.
\end{abstract}

Keywords: Design Processes; Zaha Hadid; Digital Technology; Contemporary Architecture

\section{INTRODUÇÃO}

O artigo tem como enfoque a conceituação de uma análise projetual a partir da categorização de 'modos de operar' que em sua constituição questionam a articulação entre a espacialidade do edifício, as características programáticas e a relação com seu contexto de inserção - imediato ou setorial; urbano ou natural.

A elaboração dos diagramas de leitura projetual que compõem o artigo, tem como objetivo possibilitar o estudo de projetos do percurso experimental realizado pela arquiteta Zaha Hadid ao longo de suas obras, em sua complexidade de abstração e inovação formal e material.

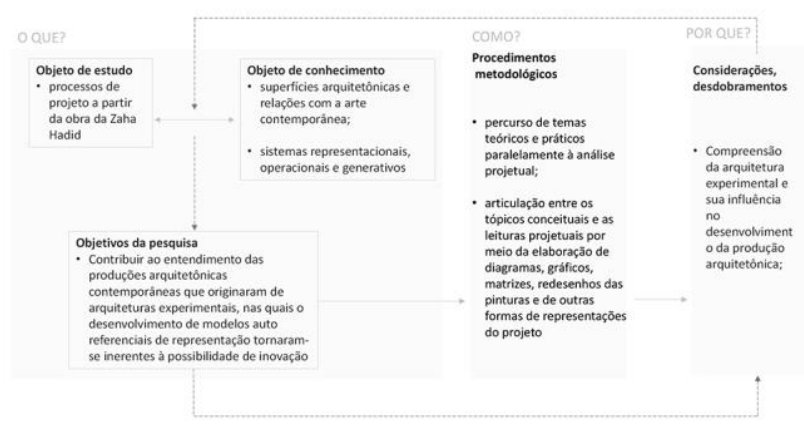

Figura 1: fluxo conceitual da pesquisa em que se situa 0 artigo. (autores)

O artigo é contextualizado em uma pesquisa mais ampla que aborda a arquitetura da Zaha Hadid, por meio de um percurso de suas obras que permite a sua leitura como uma prática e pesquisa experimental. (Fig. 1)
O mapeamento desse percurso, iniciou-se com o enfoque na importância que a superfície em seus desdobramentos espaciais, adquiriu na produção arquitetônica contemporânea, pelos meios digitais. A condição superficial da arquitetura, ocasionou o deslocamento de sua concepção como um sistema estático de relações, para um contexto de interações de dinâmicas conceituais. Catalisando o desenvolvimento de novas tectônicas, por meio da redefinição dos seus elementos constituintes. A superfície é redefinida como elemento híbrido, e de articulação espacial, formal e programática. Planos horizontais e verticais se fundem em um envelope, que se desdobra no espaço, muitas vezes, constituindo uma espacialidade curvilínea em contínua variação (Fig. 2) (Greg Lynn, 2000).

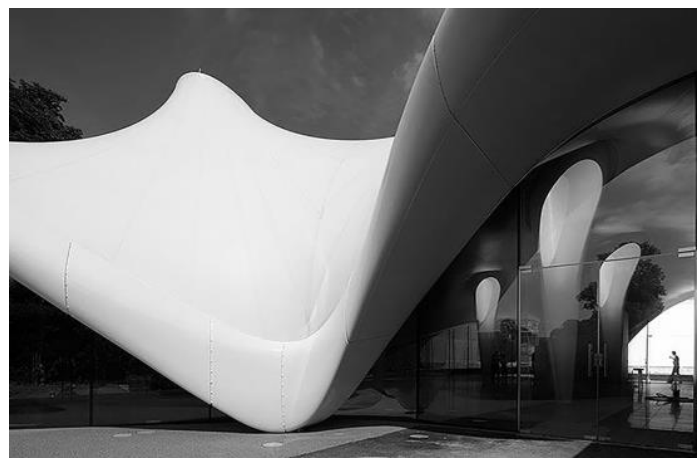

Figura 2: Fotografia externa Serpentine Sackler Gallery, ZHA, Londres, 2009-2013. (https://www.theguardian.com/)

O desenvolvimento da espacialidade superficial do projeto resulta da articulação entre a geometria do desenvolvimento da superfície e as necessidades do pavilhão por meio da variação paramétrica` (Schumacher, 2014) 


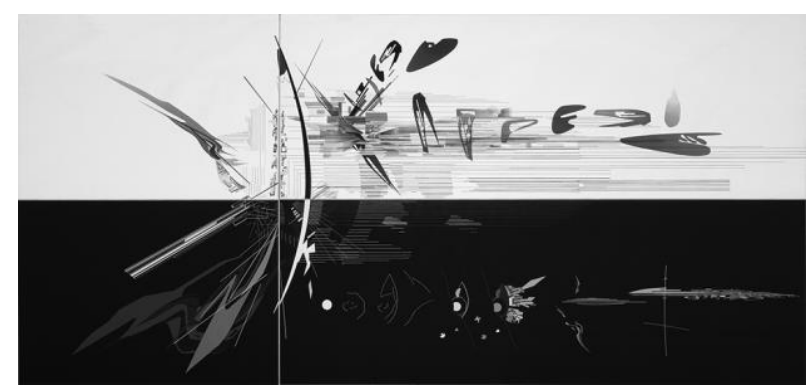

Figura 3: Pintura Visions for Madri, Zaha Hadid, 1992. (https://www.archdaily.com/798362/the-creative-process-of-zaha-hadid-asrevealed-through-her-paintings )

A superfície é abordada em dois momentos da arquitetura da Zaha Hadid. No primeiro, como plano bidimensional que define um espaço gráfico e pictórico de criação e mediação entre o conceito projetual e seu desenvolvimento. No segundo, como elemento de composição da expressão tectônica de suas obras, por meio da complexidade formal que leva ao questionamento a planaridade e ortogonalidade como características da articulação espacial e programática.

Sua exploração por meio da pintura, atua como modelo de um sistema projetivo, definido pela influência da arte Suprematista, em específico pelos trabalhos de Malevich. Hadid retoma e reconfigura elementos da arte Suprematista, Construtivista e Futurista. Iniciando um processo de criação que se desdobra em uma espacialidade que rompe com o espaço homogêneo característico da geometria Euclidiana. Resultando em uma espacialidade vetorial, em camadas, coincidente com a busca de linhas estruturais do tecido urbano, ou da paisagem natural. (Fig. 3)

O artigo procura mapear o desenvolvimento formal, espacial e programático da obra da arquiteta por intermédio da formação de matrizes diagramáticas. Esta são definidas por deslocamentos, tensões e expansões conceituais da arquitetura em direção à arte e à paisagem.

'In terms that echo Krauss's sculptural field, then, we may find combinations of architecture and landscape, architecture and biology, architecture and program, architecture and architecture producing new versions of the 'not-landscape' and the 'notsculpture' that is nonetheless 'not-exactly-architecture'. ... as we have experienced architecture up to the present' (Anthony Vidler, 2008)

\section{METODOLOGIA}

Como metodologia, o artigo adotou um recorte conceitual da pesquisa em desenvolvimento na pós-graduação, que se refere às etapas de leitura dos processos projetuais da obra 'Estação de Bombeiros Vitra' (ZHA, 1990-1994).

A obra foi escolhida por sua singularidade, dentro do percurso projetual da ZHA. Singularidade definida pelas possibilidades de correlação com outras obras sequenciais, anteriores ou posteriores. Também, pelas expansões conceituais que possibilita, em direção à outras áreas do conhecimento.
A 'Estação de Bombeiros Vitra', enquanto paisagem, considerando a relação que estabelece com 0 seu contexto, desdobra-se em duas obras subsequentes: Landscape Formation, 1996-1999, e Car Park and Terminus Hoenhm-Nord, 1998-2000. As similaridades da relação da obra e a paisagem não foram estabelecidas pela semelhança da configuração formal, mas sim, pela estratégia projetual, na qual a paisagem atua como generativa de aspectos projetuais. (Fig. 4)

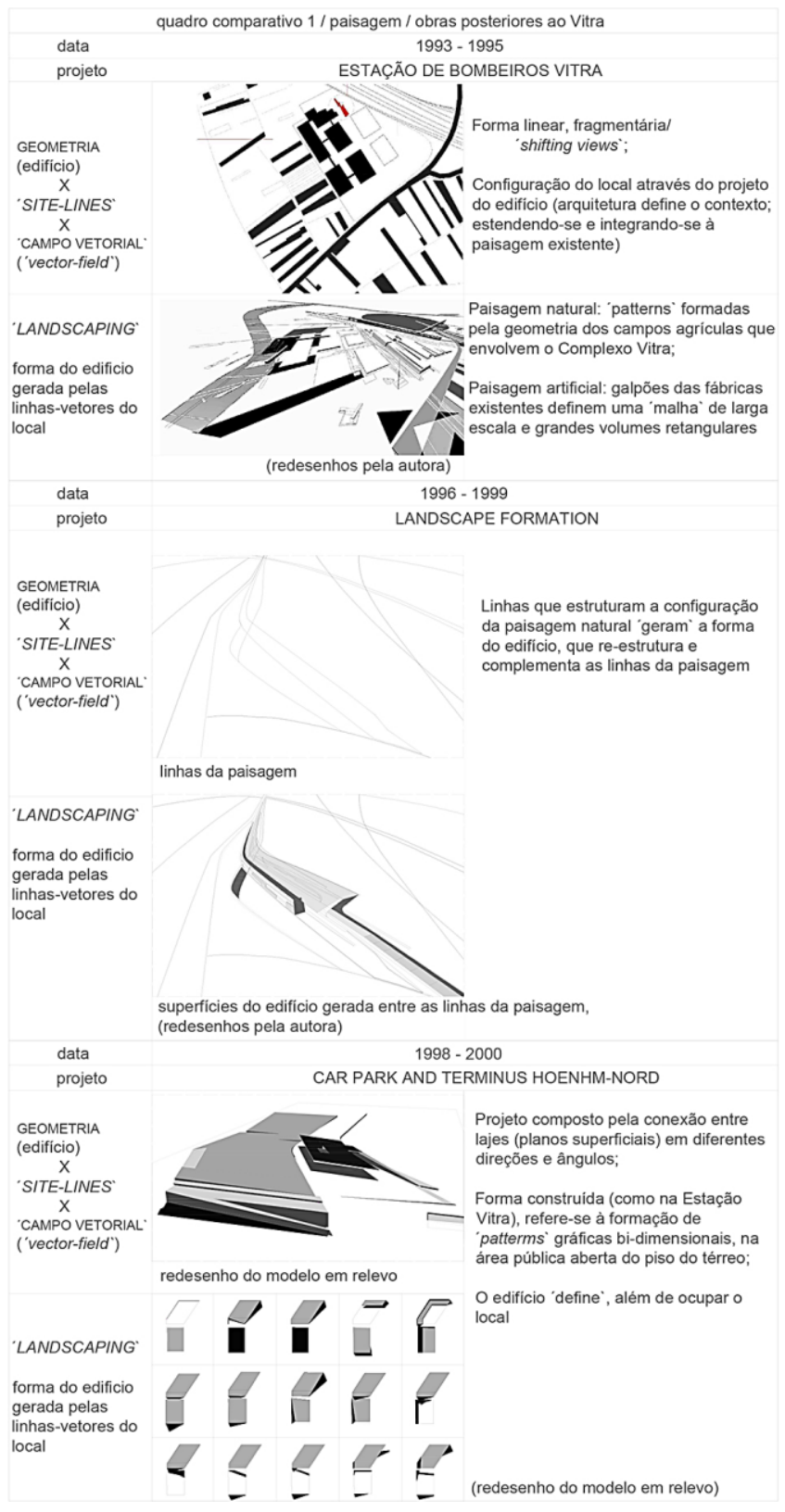

Figura 4: Quadro comparativo 1: arquitetura x paisagem. (autores)

A metodologia de leitura é desenvolvida em três etapas:

1‥ Definição dos fundamentos dos 'conceitos formativos', (Clark \& Pause, 2006), ou, categorias operativas; que estabelecem os parâmetros de leitura processual das obras;

2․ Diagramas matriciais dos conceitos formativos da 'Estação de Bombeiros Vitra', 1990-1994. Os conceitos 
formativos desdobram-se nos diagramas matriciais, formulados na leitura dos processos de projeto;

3․ Diagramas matriciais da 'Condição de Campo Ampliado` (Stan Allen, 1997, Rosalind Krauss, 1976), desdobramentos conceituais.

As matrizes das condições de campo ampliado articulam diferentes influências teórico-projetuais. Compostas principalmente pelos conceitos de 'condição de campo' (Allen, 1997) e 'campo ampliado' (Krauss, 1976, Vidler, 2002). Desenvolvido pelo arquiteto Stan Allen ('From objeto to field condition', 1997), o conceito de 'condição de campo' na arquitetura, é rebatido nas categorizações e agrupamentos das obras da ZHA por Patrik Schumacher (2002). Estabelecendo correlação com variações do diagrama do grupo Klein, desenvolvido por Rosalind Krauss no texto 'Sculpture in the Expanded Field (1976), in 'Retracing the Expanded Field' (2013), e 'The Optical Unconscious' (1996).

\section{FUNDAMENTOS}

'Foucault gives it its most precise name: it is a 'diagram', that is to say a 'functioning abstracted from any obstacle (...) or friction (and which) must be detached from any specific use. The diagram is no longer an auditory visual archive but a map, a cartography that is coextensive with the whole social field. It is an abstract machine. ', (Gilles Deleuze, Foucault, 1988, in 'Animate Form', Greg Lynn, 1999)

Os fundamentos das leituras dos processos projetuais abrangem diferentes fases de definições. Estas se iniciam na formulação de um sistema de representação generativo por meio da pintura; pela qual esse sistema tornou-se imbricado na concepção de diferentes obras da arquiteta, independentemente de sua ordem cronológica.

O entendimento da formulação do sistema de representação da Zaha Hadid, permeia as diferentes fases de sua produção.

Iniciando-se com a leitura em detalhe da pintura Suprematista, e modelos arquitetônicos de Kasimir Malevich.

Retirando parte da estética Futurista com enfoque na escultura de Umberto Boccioni.

Retendo características da pintura de Wassily Kandinsky, em aspectos da construção formal, dos grandes planos sequências nas representações pictóricas dos projetos urbanos.

Reutilizando aspectos das esculturas-relevo de Vladimir Tatlin, em sua influência nas representações por meio de 'modelos em relevo' (bas-reliefs); como análise da construção formal das superfícies em sequências de profundidade em relação à topografia da implantação do edifício.

\section{O DIAGRAMA GENERATIVO}

Adotou-se o conceito de diagrama como conceitual e generativo da arquitetura. Tendo como referência as seguintes conceituações de leituras diagramáticas, em configuração matricial:

- Diagramas Descritivos:

Descrevem o projeto sem referência à parâmetros de análise. Pode ser equivalente a um método de representação descritivo. Por exemplo, pelas projeções ortogonais do objeto arquitetônico em plantas, cortes e elevações;

- Diagramas Processuais:

Os diagramas processuais compõem o enfoque da pesquisa na qual se situa o artigo. Compõem-se dos processos de concepção arquitetônicos. Podendo fazer parte das diferentes etapas de desenvolvimento do projeto; dos primeiros croquis aos protótipos de fabricação digital;

- Diagramas Teóricos-projetuais:

Os diagramas teóricos projetuais estabelecem conexões projetivas entre as diferentes categorias de diagramas citadas acima, e conceitos tradicionalmente pertencentes ao campo teórico da arquitetura, ou à outras áreas disciplinares.

O enfoque compreende que a arquitetura é gerada pela confluência interdisciplinar e subsequente formações conceituais a partir de diagramas matriciais. Considerando que estes, estabelecendo relações de contiguidade, hibridação e diferença, entre as diferentes obras escolhidas; geram parâmetros de leituras processuais por meio da interpretação das mútuas influências de suas leituras. Essas 'mútuas influências' aproximam obras de artistas e arquitetos, colocadas em um eixo matricial $x$ e $y$, de quatro quadrantes, sendo que nas adjacências são definidos pares de correlações que geram os 'parâmetros formativos` da leitura processual em questão.

Os parâmetros formativos são definidos por categorias de diagramação processual: categorias operativas; estruturais e formativas. (Fig. 5) (Fig. 6)

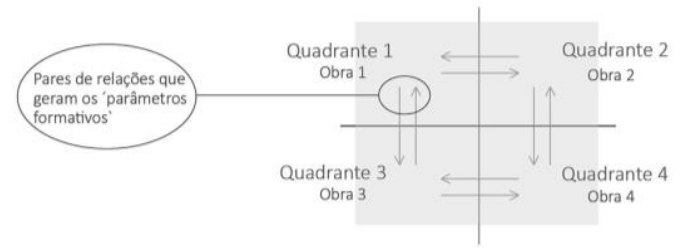

Figura 5: Diagrama 1. (autores)

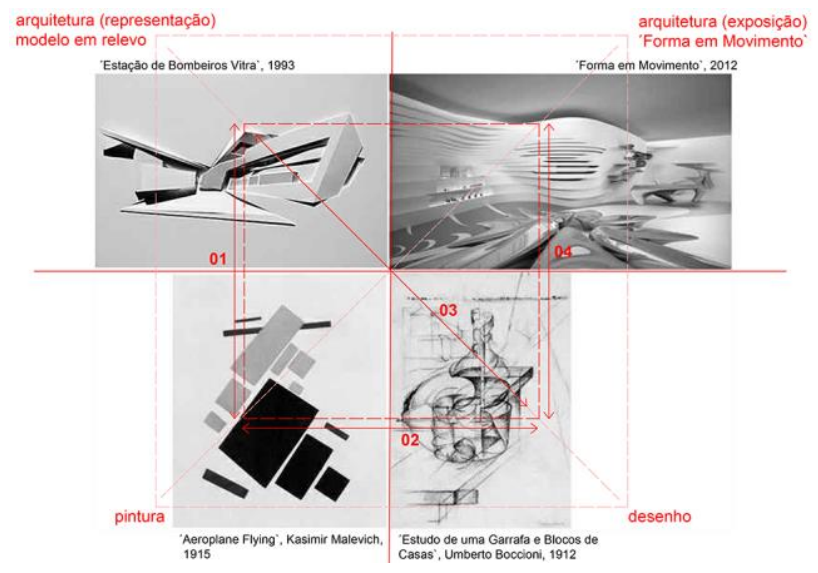

Figura 6: Diagrama 2. (autores)
3 


\section{MATRIZES CONCEITUAIS NO CAMPO AMPLIADO}

'A filosofia da desconstrução desenvolvida por Derrida tem nos permitido criticar o pensamento binário e entender como as relações hierárquicas geralmente denominadas a dois termos em pares não é natural ou pré-determinada, mas uma construção social que pode ser alterada de acordo como nos posicionamos. Em um modelo binário, tudo que um pode ser, o outro não é. Portanto, limitando a possibilidade de pensar em dois termos conjuntos. Tal modelo opera hierarquicamente, sendo um dos dois termos colocado em posição dominante. O projeto de Derrida tem por objetivo, expor a maneira com que sistemas binários permitem que as coisas sejam apenas 'igual' ou 'diferente' à categoria dominante e redefine tais normas intelectuais predominantes com novas formulações', (Rendell, 2006, p.9)

As matrizes apresentadas no artigo têm como referência os conceitos desenvolvidos em dois textos seminais dos anos 1970 e 1990: 'The Sculpture in the Expanded Field' (Rosalind Krauss, 1976) e 'Field Conditions' (Stan Allen, 1997). Textos que após décadas, em diferentes releituras, contribuem com a conceituação da inclusão digital na arquitetura. E, também, com a revisão dos conceitos formativos de uma arquitetura 'tipológica', para o enfoque nos processos projetuais de criação. O texto de Stan Allen 'Field Conditions` (1997), foi republicado na coletânea organizada por Mario Carpo in 'The Digital Turn in Architecture 1992-2012 em 2013.

$\mathrm{O}$ artigo considera que a arquiteta Zaha Hadid, opera a concepção e desenvolvimento projetual, por meio de matrizes conceituais em uma relação de campo, evitando a formação de pares binários de oposição conceitual. A condição de campo (Stan Allen, 1997), possibilita a formação conceitual da condição de assemblage dos elementos projetuais, em infinitas configurações. Organizações sistêmicas em interações contínuas, por meio de sua estratificação em camadas sobrepostas. Arquitetura pensada como mediação - considerando como referência a tese de Schumacher sobre a relação entre forma e medium:

'Forms are always forms formed within a medium, and a medium is a medium for a certain type of forms only as long as it allows for the ongoing formation of these forms. A paradigmatic example: a sentence $(=$ form $)$ is a strict coupling of words selected from the loosely coupled set of words given by the vocabulary of the respective language (= medium). In this sense definite, individual drawings and digital models are 'forms', i.e. strict couplings, selected from the loosely coupled elements (points, lines, graphic objects, CAD primitives) of the design medium of drawing/ digital model. `(Patrik Schumacher, 2010)

O conceito de campo ampliado de Rosalind Krauss, é entendido, como uma definição dinâmica de combinação de categorias. As relações estabelecidas significam uma tentativa de estender definições (Rendell, 2006) préestabelecidas e de articular conjuntos de relações.

As camadas articuladas através do 'quadrado semiótico do Grupo de Klein`são (Rendell, 2006):

1ำ Termo: contradição, ou simples negativos de dois termos dominantes;

2ำ Termo: complexo, formado pela articulação entre duas afirmações;
3ำ Termo: neutro, formado pela negação.

Foram elaboradas três variações do 'quadrado semiótico do Grupo de Klein`, considerando as leituras de Rosalind Krauss (1976) e Jane Rendell (2006).

A primeira variação, 'Condição de Campo 1; tensão superficial', refere-se à configuração original dos conceitos articulados no texto de Krauss (1993), entre figura e fundo (ground).

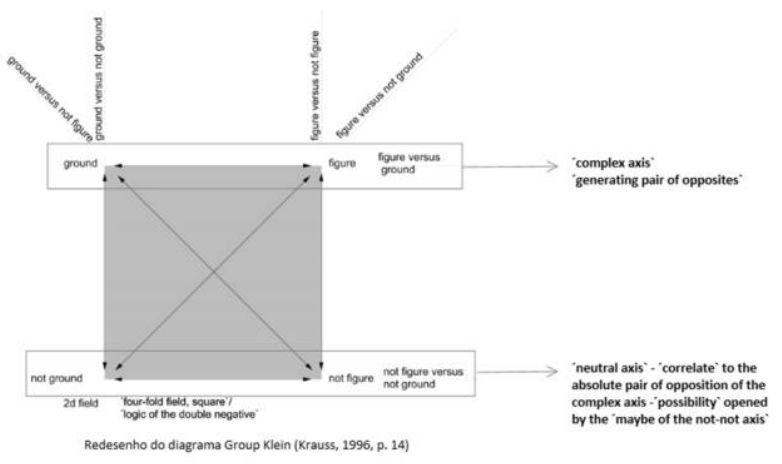

Figura 7: Diagrama 3, 'condição de campo 1'. Redesenho com algumas alterações. (Krauss, 1996, p. 14)

As pinturas Suprematistas de Malevich, através do quadrado semiótico de Klein-Krauss, podem ser entendidas pela apreensão semiótica de termos opostos, que geram múltiplas possibilidades de variação através da relação entre fundo $x$ figura $x$ perímetro.

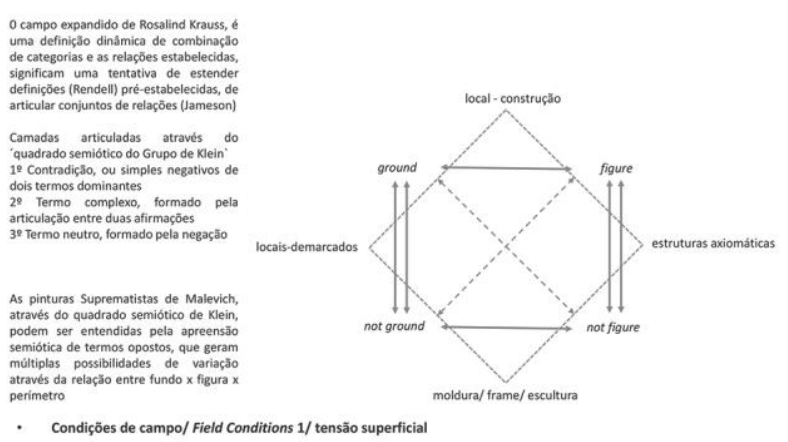

Figura 8: Diagrama 4, 'condição de campo 1'. Redesenho com algumas alterações. (Krauss, 1996, p. 14)

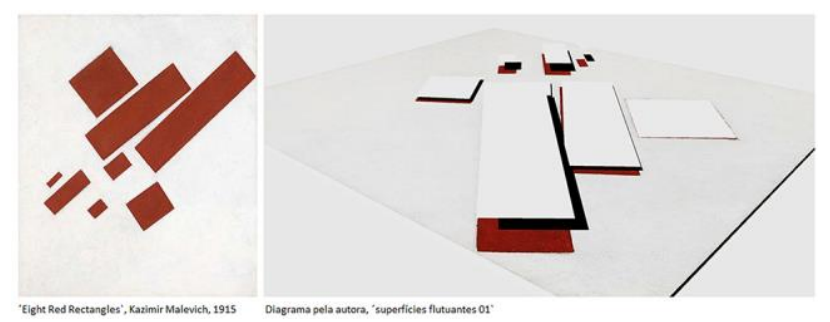

Figura 9: (esquerda) 'Eight red rectangles', Malevich, 1915 (https://en.wikipedia.org/wiki/Kazimir_Malevich), redesenho da pintura de Malevich, em vista perspectivada.

A segunda variação, 'Condição de Campo 2; rotação de planos', refere-se ao sistema projetivo por meio da pintura na arquitetura da Zaha Hadid. Os dois termos dominantes são formados pelo 'plano da pintura' ('ground), e 'pintura'. 
O projeto citado é o Car Park and Terminus HoenhmNord, 1998-2000. O qual é analisado por Hal Foster (2015), sob o ponto de vista da 'rotação dos planos', que considera o tratamento gráfico do piso térreo, como plano pictórico na posição horizontal.

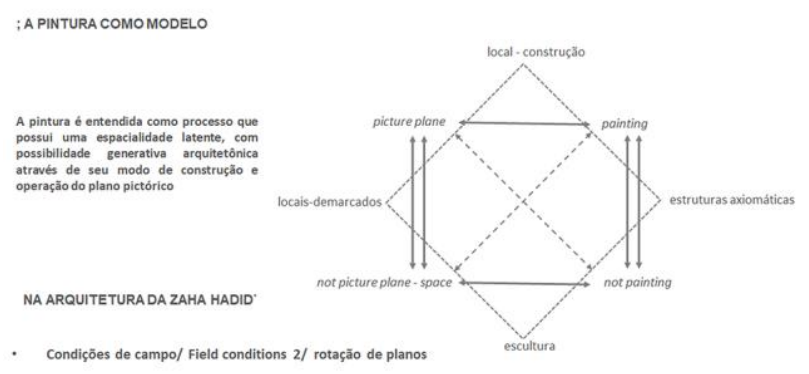

Figura 10: Diagrama 5, 'condição de campo 2'. Redesenho com algumas alterações. (Krauss, 1996, p. 14)

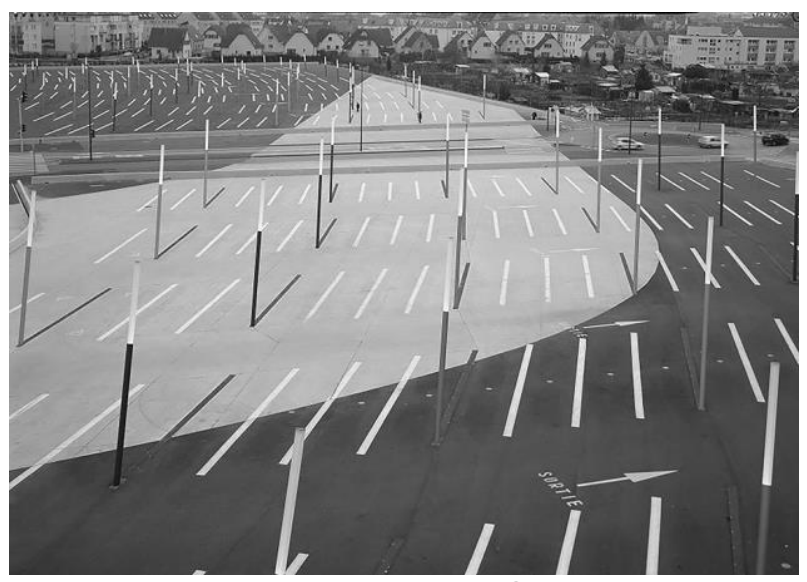

Figura 11: fotografia estacionamento Car Park and Terminus Hoenhm-Nord, ZHA, 1998-2000. (http://www.zaha-hadid.com).

Hadid atua no intervalo entre a arquitetura e a escultura, adotando a paisagem imediata como 'campo' vetorial/ processual de formação de possibilidades projetuais (Stan Allen, 1999).

A arquitetura pensada como um processo, desloca o enfoque tradicional da disciplina na idealização a priori de uma forma e espacialidade totalizante, inerte às mudanças contextuais de seu entorno, de sua interioridade e principalmente, das contingências naturais de uma continuidade histórica à qual é submetida em sua condição de existência em um intervalo temporal. Existência matérica, informacional ou midiática, que ocorre em modulações temporais. A arquitetura tornou-se 'processo', em sua concepção e em sua existência. Denominações dispensáveis, já que em seu estado processual, apenas estabelece 'modos' de existência, seja através de uma sequência de codificação algorítmica, na qual encontrará infinitas variações, como infinitas formas de materialização, na modulação temporal de sua existência. Se for efêmera, como uma alteração de densidade entre estados de transição da matéria, torna-se plasmável aos corpos imersivos. O caráter objetual dissipa-se em fragmentos processuais, interligados pelas contingências contextuais, que se apresentam como pontos de saída da lógica processual; local, lugar, ambiente, intervalo, situação, estado plasmável ou 'fôrmas do visível'.
A arquitetura em processo, ocasiona a revisão de seu sistema projetivo, já que não encontra mais na espacialidade homogênea da geometria cartesiana, o seu referente. Necessita da espacialidade topológica, de coordenadas variáveis, de linhas vetoriais, de superfícies plasmáveis que se alteram em profundidade por modulações temporais. A duração temporal tornou-se sua unidade de medida. Dimensão da matéria em diferença de viscosidade. $O$ evento programável, sua performance matérica desenvolve-se em estado de coexistência com os corpos imersivos. Ocupar o espaço é moldá-lo ao corpo em movimento ou em repouso. O conceito de 'complexo', que remete à 'compósito' (Hal Foster, 2015) é levado ao extremo. Complexo arte-arquitetura, processo-matéria, evento-imagem, evento-performance dos corpos no espaço.

Hal Foster, no livro 'O Complexo Arte-Arquitetura', comenta sobre a fase de formação conceitual no final dos anos 1970 de várias práticas arquitetônicas:

'Também nessa época, arquitetos como Frank Gehry tinham desenvolvido a criação de formas dos projetos de vanguarda a tal ponto que estes tiveram que defrontar (mais uma vez) seu próprio dilema modernista: como dada essa liberdade aparente, fundamentar as decisões arquitetônicas? Foi em grande parte uma preocupação com os modos de representação o que poupou Eisenman e Hadid das deliberadas transformações da forma de Gehry e seus seguidores. ' (Foster, 2015, p.100)

Do objeto à condição de campo / o minimalismo e a condição de campo (Allen, 1997)

A condição de campo, antes de possibilitar uma aproximação direta com as artes visuais, traz para o interior da produção arquitetônica sua liberação dos contingentes do objeto como forma totalizadora e unitária.

O enfoque inovador do texto, define a sua influência na produção arquitetônica até a atualidade. A utilização de recursos computacionais, segundo o arquiteto Greg Lynn, ocasiona a imersão do processo de concepção arquitetônica em um meio dinâmico, que possibilita a simulação de forças contextuais no ambiente virtual, do meio computacional.

A terceira variação, 'Condição de Campo 3; arquitetura como paisagem ', refere-se ao sistema projetivo por meio da paisagem. Esta, considerada elemento generativo da arquitetura. Os dois termos dominantes são formados pela 'paisagem ' ('ground), e 'arquitetura'.

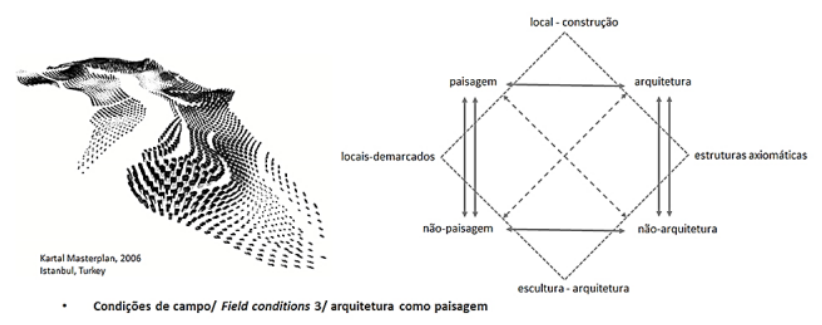

Figura 12: Diagrama 6, 'condição de campo 3'. Redesenho pelos autores, com algumas alterações. (Krauss, 1996, p. 14) 


\section{MATRIZES CONCEITUAIS NA OBRA 'ESTAÇÃO DE BOMBEIROS VITRA}

A leitura da Estação de Bombeiros Vitra é dividida em dois enfoques principais, o edifício e a paisagem; a interioridade da arquitetura (Eisenman, 1999) e a arquitetura como paisagem, 'landscaping'.

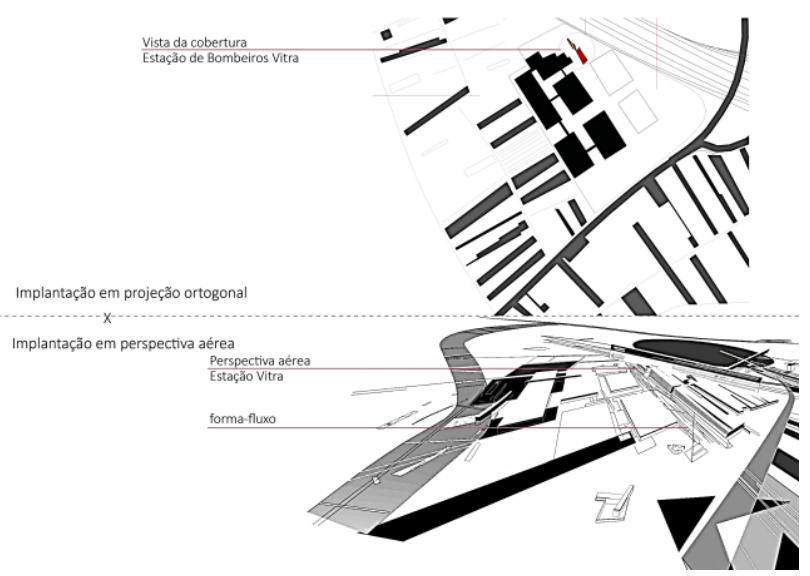

Figura 13: implantação Complexo Vitra (redesenho 'pelos autores). A figura é composta por duas formas de projeção da implantação. Uma vista em planta do complexo Vitra, circundado pela paisagem dos campos agrícolas. E uma vista do modelo tridimensional da implantação, redesenhado a partir da pintura da vista aérea do Complexo Vitra (Zaha Hadid, 1995).

O objetivo é o entendimento da relação estabelecida entre obrapaisagem e visualidade, nos estudos projetivos-pictóricos executados pela arquiteta.

Esta leitura processual, situa-se nas matrizes da 'Condição de campo 3' (Fig. 12). A articulação entre os dois termos dominantes, paisagem e arquitetura, acontece pela projeção.

What would it be like to be in a space capable of resembling frozen movement? A space that seems to be in movement but that is also a projection, a frozen instant.'(Zaha Hadid, 1995, p. 6)

O estudo da implantação, pelos autores, procura estabelecer as hibridações em processo, dos métodos de representação e de concepção projetual.

A importância de explorar a pintura como método projetivo da arquitetura, permeia o enfoque da leitura da obra. Adquirindo mais importância para o entendimento dos processos envolvidos na concepção projetual, do que as formas tradicionais de representação. Da mesma forma que a arquiteta cita a importância da escolha da pintura como meio projetivo, pela possibilidade de desenvolvimento do próprio processo projetual, é na pintura, pelo entendimento dos autores, que se situa o entendimento de sua obra.
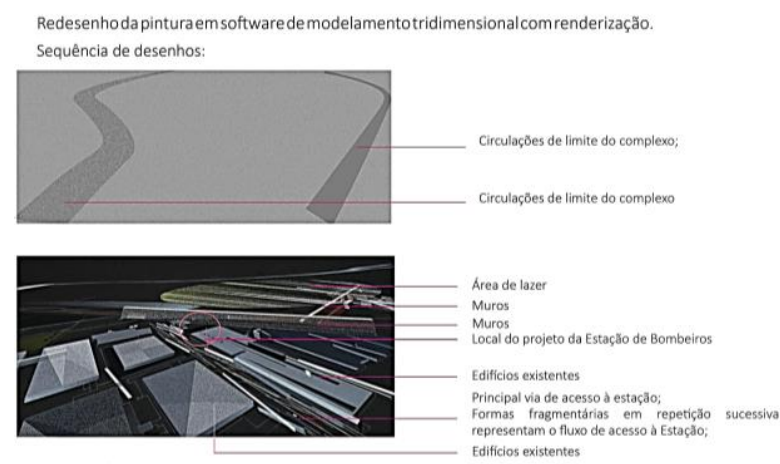

Figura 14: etapas de redesenho da implantação do Complexo Vitra. (autores)
A partir das figuras geradas no redesenho da pintura, que tornou-se um modelo tridimensional, em escala e com precisão (devido ao método de construção da pintura pela Zaha Hadid) foram geradas várias imagens, por meio de linhas ou de renderização das superfícies, possibilitando a análise de aspectos formativos, como a identificação das diferentes malhas que se sobrepõem, criando uma dinâmica de diferentes eixos, e vetores, resultando no conceito forma-fluxo adotado na leitura. (Fig. 15 a 18)

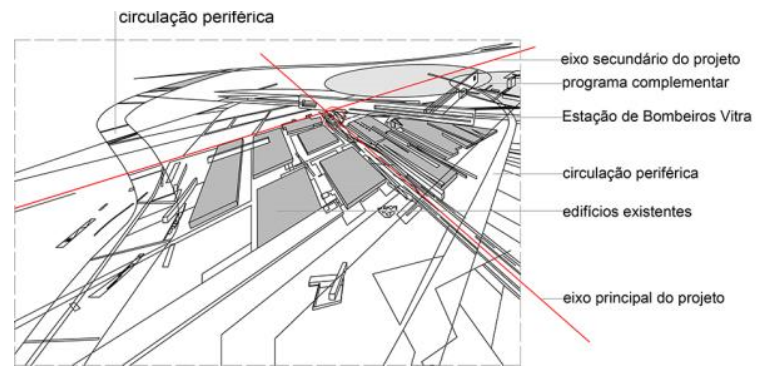

Figura 15: etapas de redesenho da implantação do Complexo Vitra; eixos de circulação e distribuição do programa. (autores)
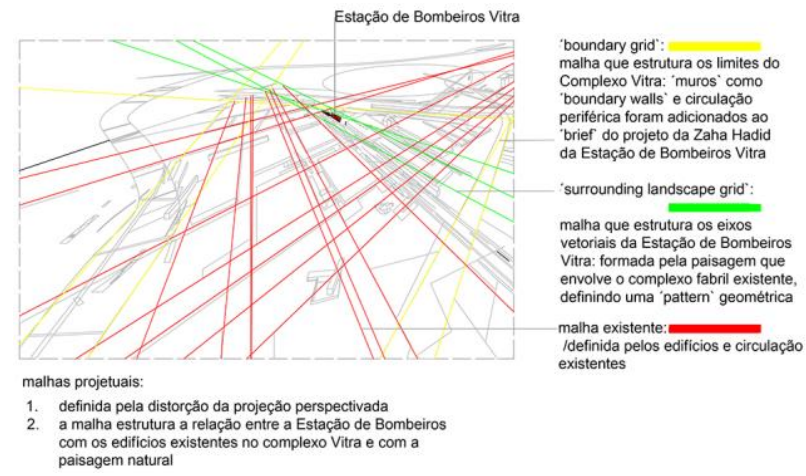
existentes a malha estrutura a relaçăo entre a Estaçăa de Bombeiros
com os edificios existentes no complexo Vitra e com a

Figura 16: etapas de redesenho da implantação do Complexo Vitra; malhas contextuais da implantação da Estação de Bombeiros. (autores)

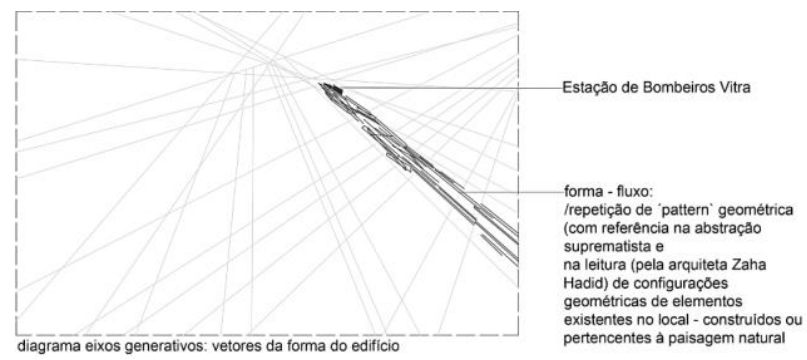

Figura 17: etapas de redesenho da implantação do Complexo Vitra; 'forma-fluxo'. (autores)

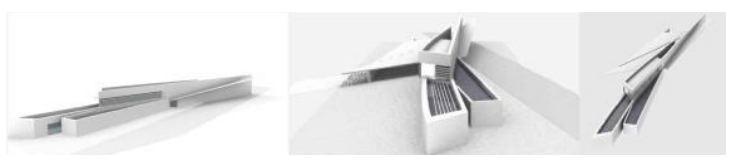

Figura 18: diferentes vistas em perspectiva. (autores)

\section{RESULTADOS}

Os resultados esperados referem-se à compreensão de processos de projeto na contextualização atual dos meios digitais e das diferentes fases de inclusão digital na qual a produção arquitetônica contemporânea tem se desenvolvido. 
Foram concluídos dois conceitos relativos à leitura e diagramas da estação Vitra: o conceito de 'campos digitais'e de 'paisagens generativas'.

Os campos digitais estão relacionados à leitura do objeto arquitetônico em sua condição de campo, que objetiva a identificação da matriz conceitual que gera a sua configuração e articulação sistêmica. Isto é, o objeto é uma configuração sistêmica estável, emergente da condição de campo.

A conclusão seguinte, refere-se ao conceito de 'paisagens generativas'. A importância da paisagem como sistema de dinâmica temporal, que catalisa a formação projetual.

Nas duas conclusões está subentendida a inclusão digital na arquitetura. Já que as relações recíprocas nos campos digitais e nas paisagens generativas são equacionadas pelo meio digital.

\section{DISCUSSÃO}

As contribuições para a área do conhecimento referem-se aos seguintes tópicos:

1. Mapear a interdisciplinaridade na produção arquitetônica contemporânea a partir de obras da arquiteta Zaha Hadid;

2. Investigar as possibilidades de interação entre a pesquisa acadêmica e a prática arquitetônica;

3. Desenvolver uma metodologia de análise gráfica por meio de diversas modalidades de diagramas conceituais, operativos e representacionais;

4. Compreender as produções arquitetônicas contemporâneas que originaram de arquiteturas experimentais, nas quais o desenvolvimento de modelos auto referenciais de representação tornaram-se inerentes à possibilidade de inovação.

\section{AGRADECIMENTOS}

O presente trabalho foi realizado com apoio da Coordenação de Aperfeiçoamento de Pessoal de Nível Superior - Brasil (CAPES) - Código de Financiamento 001.

\section{REFERÊNCIAS}

Allen, S. (1999). Points + Lines Diagrams and Projects for the City. Nova York: Princeton Architectural Press.

Architect, G. (1986). Zaha M. Hadid. Tóquio: A.D.A. Edita Tokyo.

Carpo, Mario (org.) (2013). The Digital Turn in Architecture 1992 2012. Willey (AD Reader, $1^{\text {st }}$. edition ebook).

Clark, Roger H. \& Pause, Michael. (2006). Precedents in architecture. Analytic diagrams, formative ideas, and parti. New Jersey: John Willey \& Sons.

Eisenman, Peter \& Somol, Robert (1999). Peter Eisenman, Diagram Diaries. Cambridge: Universe Publishing.

Fontana-Giusti, G., \& Shumacher, P. (2004). Process: Sketches and Drawings. Londres: Thames and Hudson.

Fontana-Giusti, G., \& Shumacher, P. (2004). Texts and References. Londres: Thames and Hudson.

Foster, H. (2015). O Complexo Arte-Arquitetura. CosacNaify: São Paulo.

Hadid, Z. (1998). Zaha Hadid, The Complete Buildings and Projects. Londres: Thames and Hudson Itda.

Hadid, Z. \& Rojo, Luis C. (1995). Zaha Hadid 1992-1995. Conversation with Zaha Hadid, El Croquis. Barcelona: Ivory Press.

Krauss, R. (1993). The Optical Unconscious. Cambridge: The MIT Press.

Lynn, Greg. (1998). Animate Form. Princeton: Princeton Architectural Press.

Papapetros, Spyros \& Rose, Julian (2013). Retracing the Expanded Field, Encounters between Art and Architecture. Cambridge: The MIT Press.

Pons, J. P. (2002). Neovanguardias y Representación Arquitectónica. Barcelona: Edicions de la Universitat Politècnica de Catalunya UPC.

Rendell, Jane (2006). Art and Architecture: A place between. I. B. Tauris: Londres.

Sykes, A. K. (2013). O Campo Ampliado da Arquitetura: Antologia Teórica 1993-2009. São Paulo: Cosac Naify.

i Clark \& Pause in 'Precedents in Architecture, analytic diagrams, formative ideas, and partis' (2005, p. 235), desenvolve o conceito de 'formative ideas '. No artigo é empregado o termo 'conceitos formativos' em substituição a 'formative ideas':

'A formative idea is understood to be a concept which a designer can use to influence or give form to design. The ideas offer ways to organize decision, to provide order, and to consciously generate form. By engaging one formative idea instead of another, a designer begins to determine the formal result and the manner in which it will differ from other configurations. The use of different ordering ideas may generate different results. ', (p. 235) 